\title{
Beamspace matrix pencil method for direction of arrival estimation
}

\author{
Muhammad Faisal Khan ${ }^{\mathrm{a})}$ and Muhammad Tufail \\ Department of Electrical Engineering, Pakistan Institute of Engineering and \\ Applied Sciences, Islamabad 45650, Pakistan \\ a)phd0514@pieas.edu.pk
}

\begin{abstract}
In this paper, we propose DFT Beamspace Matrix pencil (BMP) method for direction of arrival estimation (DOA). The DFT beamspace approach has the advantage of reduced computational complexity due to converting complex computations (in Matrix Pencil method) into real ones along with the inherent ability to operate in a reduced beam-space in situations where some priori information is available about the DOAs. Furthermore, a novel Multiple Invariance BMP (MBMP) method is also developed and it is shown through simulations that its reduced beamspace version has comparable performance to the existing Unitary Matrix Pencil method (UMP) with lower computational cost.
\end{abstract}

Keywords: matrix pencil, DFT, beamspace, DOA estimation

Classification: Science and engineering for electronics

\section{References}

[1] T. K. Sarkar and O. Pereira, "Using the matrix pencil method to estimate the parameters of a sum of complex exponentials," IEEE Antennas Propag. Mag., vol. 37, no. 1, pp. 48-55, Feb. 1995.

[2] N. Yilmazer, J. Koh, and T. K. Sarkar, "Utilization of a unitary transform for efficient computation in the matrix pencil method to find the direction of arrival," IEEE Trans. Antennas Propag., vol. 54, no. 1, pp. 175-181, Jan. 2006.

[3] P. Stoica and A. Nehorai, "Comparitive performance study of elementspcae and beam-space MUSIC estimators," Circuits, Syst. Signal Process., vol. 10, no. 3, pp. 285-292, Nov. 1991.

[4] M. D. Zoltowski, M. Haardt, and C. P. Mathews, "Closed-form 2-D angle estimation with rectangular arrays in element space or beamspace via unitary ESPRIT," IEEE Trans. Signal Process., vol. 44, no. 2, pp. 316328, Feb. 1996.

[5] T. Ferreira, S. L. Netto, and P. S. R. Diniz, "Beamspace covariance-based DOA estimation," Proc. Int'l Workshop on Signal Processing Advances for Wireless Communications (SPAWC), pp. 136-140, 2008.

[6] G. H. Golub and C. F. Van Loan, Book Matrix Computations 3rd. ed., The Johns Hopkins Univ. Press, Baltimore, 1996. 


\section{Introduction}

The Matrix Pencil (MP) method [1] is a popular high resolution method which requires only one snapshot for estimating DOA in real time nonstationary environment. Its real time implementation, requires minimum computational burden. The most computationally expensive part of MP method is singular value decomposition (SVD) of a complex data matrix. In [2], a UMP method was developed which reduces the computational effort to about $\frac{1}{4}$ by converting complex data matrix into a real matrix. If some priori information about the DOAs is available, as in radar applications, then reduced dimension processing of data is possible as in beamspace MUSIC, ESPRIT and CB-DOA methods [3, 4, 5]. These methods, however, are multi-snapshot covariance based algorithms and requires additional spatial smoothing in situations where incoming signals are fully correlated.

In this paper, we propose a (single-snapshot) Beamspace Matrix Pencil (BMP) method which transforms the complex data matrix in MP method into a real and reduced dimensional matrix using selected rows of a DFT matrix. The rows which are selected to estimate DOA in a sector of interest are based upon a priori information. Depending upon the number of rows selected, the computational burden is decreased several times. If there is no a priori information then BMP method can be applied via parallel processing with overlapped sectors [4]. We also introduce a Multiple Invariance BMP (MBMP) method whose performance is comparable with UMP method and computational complexity is almost half.

\section{A brief review of MP and UMP methods}

If $I$ signals of wavelength $\lambda$ are impinging on a uniform linear array (ULA) of $N$ sensors with angles $\theta_{i}$ then voltage measured at $n$th sensor can be expressed as

$$
y(n)=x(n)+\nu(n)=\sum_{i=1}^{I} R_{i} z_{i}^{n}+\nu(n)
$$

where $x(n)$ is actual signal voltage, $\nu(n)$ is the noise signal at the $n$th sensor, $R_{i}$ is the complex amplitude of $i$ th signal and $z_{i}=\exp \left(j \frac{2 \pi}{\lambda} d \cos \theta_{i}\right)$. Here, $d$ is the distance between two consecutive sensors. A data matrix $Y$ can be constructed as

$$
Y=\left[\begin{array}{cccc}
y(0) & y(1) & \cdots & y(L) \\
y(1) & y(2) & \cdots & y(L+1) \\
\vdots & \vdots & \ddots & \vdots \\
y(N-L-1) & y(N-L) & \cdots & y(N-1)
\end{array}\right]_{(N-L+1) \times(L)}
$$

where $L$ is the pencil parameter [1]. To estimate DOA in MP method, the SVD of $Y$ is needed, which requires $O\left\{(N-L+1)^{3}\right\}$ complex multiplications. In UMP method, $Y$ is transformed into a real matrix, which reduces the computational complexity to about $1 / 4$ of MP method. 


\section{Beamspace matrix pencil method}

The noiseless data matrix in MP method can be written as

$$
Y=Z_{a} R_{o} Z_{b}
$$

where $z_{a i}\left(i\right.$ th column of $\left.Z_{a}\right), z_{b i}\left(i\right.$ th row of $\left.Z_{b}\right)$ and $R_{o}$ are defined as

$$
\begin{aligned}
z_{a i} & =\left[e^{-j\left(\frac{M-1}{2}\right) \mu_{i}}, \cdots, e^{-j \mu_{i}}, 1, e^{j \mu_{i}}, \cdots, e^{j\left(\frac{M-1}{2}\right) \mu_{i}}\right]^{T} \\
z_{b i} & =\left[1, e^{j \mu_{i}}, \cdots, e^{j L \mu_{i}}\right] \\
R_{o} & =\operatorname{diag}\left[e^{j \frac{M-1}{2} \mu_{1}} R_{1}, e^{j \frac{M-1}{2} \mu_{2}} R_{2}, \cdots, e^{j \frac{M-1}{2} \mu_{I}} R_{I}\right]
\end{aligned}
$$

where $1 \leq i \leq I, M=N-L+1$ and $\mu_{i}=\frac{2 \pi}{\lambda} d \cos \theta_{i}$. Now, if $\mathrm{W}$ is a DFT matrix of size $M \times M$ then the $m$ th row of $W^{H}$ will be

$$
w_{m}^{H}=e^{j\left(\frac{M-1}{2}\right) m \frac{2 \pi}{M}} \cdot\left[1, e^{-j m \frac{2 \pi}{M}}, e^{-j 2 m \frac{2 \pi}{M}}, \cdots, e^{-j(M-1) m \frac{2 \pi}{M}}\right] .
$$

The row vector $w_{m}^{H}$ represents a DFT beam steered at a spatial frequency $\mu=m \frac{2 \pi}{M}$. If (3) is multiplied by $W^{H}$ from left, we get

$$
Y_{w}=W^{H} Z_{a} R_{o} Z_{b}=B R_{o} Z_{b}
$$

where $B=W^{H} Z_{a}$ is a beamspace array manifold matrix of size $M \times I$ whose $m$ th element $(0 \leq m \leq M-1)$ of $i$ th column $(1 \leq i \leq I)$ can be written as

$$
b_{m}\left(\mu_{i}\right)=\frac{\sin \left[\frac{M}{2}\left(\mu_{i}-m \frac{2 \pi}{M}\right)\right]}{\sin \left[\frac{1}{2}\left(\mu_{i}-m \frac{2 \pi}{M}\right)\right]}
$$

and the relation between $b_{m}$ and $b_{m+1}$ can be shown to be [4]

$$
\begin{aligned}
\tan \left(\frac{\mu_{i}}{2}\right) & \left\{\cos \left(m \frac{\pi}{M}\right) b_{m}\left(\mu_{i}\right)+\cos \left((m+1) \frac{\pi}{M}\right) b_{m+1}\left(\mu_{i}\right)\right\} \\
& =\sin \left(m \frac{\pi}{M}\right) b_{m}\left(\mu_{i}\right)+\sin \left((m+1) \frac{\pi}{M}\right) b_{m+1}\left(\mu_{i}\right) .
\end{aligned}
$$

From (10), we get $M$ equations for $(0 \leq m \leq M-1)$, which can be written as

$$
\tan \left(\frac{\mu_{i}}{2}\right) \Gamma_{1} \mathbf{b}\left(\mu_{i}\right)=\Gamma_{2} \mathbf{b}\left(\mu_{i}\right)
$$

where

$$
\mathbf{b}\left(\mu_{i}\right)=\left[\begin{array}{llll}
b_{0}\left(\mu_{i}\right) & b_{1}\left(\mu_{i}\right) & \cdots & b_{M-1}\left(\mu_{i}\right)
\end{array}\right]^{T} .
$$

The only two non-zero elements of the $p$ th row of $\Gamma_{1}$ will be the $p$ th element which is $\cos \frac{(p-1) \pi}{M}$ and the $q$ th element which will either be $\cos \frac{p \pi}{M}$ for $(1 \leq$ $p \leq M-1 ; q=p+1)$ or $(-1)^{M+1} \cos \frac{p \pi}{M}$ for $(p=M ; q=1)$. The $p$ th row of $\Gamma_{2}$ has the similar form where cosine functions are replaced by sine functions. If we define

$$
\mathbf{B}=\left[\begin{array}{llll}
\mathbf{b}\left(\mu_{1}\right) & \mathbf{b}\left(\mu_{2}\right) & \cdots & \mathbf{b}\left(\mu_{I}\right)
\end{array}\right]_{(M \times I)}
$$

then for $I$ sources, (11) can be written in compact form as

$$
\Gamma_{1} \mathbf{B} \Omega=\Gamma_{2} \mathbf{B}
$$

where

$$
\Omega=\operatorname{diag}\left[\tan \left(\frac{\mu_{1}}{2}\right), \tan \left(\frac{\mu_{2}}{2}\right), \cdots, \tan \left(\frac{\mu_{I}}{2}\right)\right] .
$$


From the definitions of $R_{o}$ and $Z_{b}$, it is obvious that they are full rank matrices which implies that the matrices $Y_{w}$ and $\mathbf{B}$ share the same column space. For noisy data, the largest $I$ left singular vectors of matrix $\left[\operatorname{Re}\left\{Y_{w}\right\}, \operatorname{Im}\left\{Y_{w}\right\}\right]$ will span the column space of $\mathbf{B}$. If $E_{s}$ contains these left singular vectors then we have

$$
E_{s}=\mathbf{B} T
$$

where $T$ is a non-singular $I \times I$ matrix. Using (16) in (14) we can write

$$
\Gamma_{1} E_{s} \Psi=\Gamma_{2} E_{s} \Rightarrow \Psi=\left(\Gamma_{1} E_{s}\right)^{\dagger} \Gamma_{2} E_{s}
$$

where $\Psi=T^{-1} \Omega T$ and ${ }^{\dagger}$ denotes pseudo-inverse. Thus, the eigenvalues of $I \times I$ matrix $\Psi$ are $\tan \left(\mu_{i} / 2\right)$ and the required DOAs can be calculated from the equation $\mu_{i}=2 \tan ^{-1}\left\{\frac{2 \pi}{\lambda} d \cos \theta_{i}\right\}$.

The advantage of BMP method is evident when one employs a subset of $k$ rows of $W^{H}$. The selection of these rows depends upon the location and the width of sector of interest which is known from a priori information [4]. Hence, a reduced dimensional beamspace data matrix is obtained as $Y_{w}^{r}=\left[W^{r}\right]^{H} Y$ where the superscript ${ }^{r}$ is used to denote the matrix with reduced dimensions. If $E_{s}^{r}$ contains the largest $I$ left singular vectors of $\left[\operatorname{Re}\left\{Y_{w}^{r}\right\}, \operatorname{Im}\left\{Y_{w}^{r}\right\}\right]$ then the required parameters can be calculated from the eigenvalues of the matrix $\Psi=\left[\Gamma_{1}^{r} E_{s}^{r}\right]^{\dagger} \Gamma_{2}^{r} E_{s}^{r}$ where $\Gamma_{1}^{r}$ and $\Gamma_{2}^{r}$ of size $(k-1) \times k$ are appropriate sub blocks of $\Gamma_{1}$ and $\Gamma_{2}$, respectively.

\section{Multiple invariance beamspace matrix pencil method}

In (10), only the relation between $b_{m}$ and $b_{m+1}$ is considered. In more general form, we can write the relation between $b_{m}$ and $b_{m+l}(1 \leq l \leq M-1)$ as

$$
\begin{aligned}
\tan \left(\frac{\mu_{i}}{2}\right) & \left\{\cos \left(m \frac{\pi}{M}\right) b_{m}\left(\mu_{i}\right)+(-1)^{(l+1)} \cos \left((m+l) \frac{\pi}{M}\right) b_{m+l}\left(\mu_{i}\right)\right\} \\
& =\sin \left(m \frac{\pi}{M}\right) b_{m}\left(\mu_{i}\right)+(-1)^{(l+1)} \sin \left((m+l) \frac{\pi}{M}\right) b_{m+l}\left(\mu_{i}\right) .
\end{aligned}
$$

The above equation (for $l$ th invariance) can be written in the matrix form as

$$
\Gamma_{l_{1}} \mathbf{B} \Omega=\Gamma_{l_{2}} \mathbf{B}
$$

where $\Gamma_{l_{1}}$ and $\Gamma_{l_{2}}$ are appropriate selection matrices. Only the $p$ th and the $q$ th elements of $p$ th row of $\Gamma_{l_{1}}$ are non zero. The $p$ th element is $\cos \frac{(p-1) \pi}{M}$ and the $q$ th element will be either $(-1)^{l+1} \cos \frac{(p+l-1) \pi}{M}(1 \leq p \leq M-l ; q=p+l)$ or $(-1)^{M+l} \cos \frac{(p+l-1) \pi}{M}(p>M-l ; q=p+l-M)$. The $p$ th row of $\Gamma_{l_{2}}$ has the similar form where cosine functions are replaced by sine functions. In constructing $\Gamma_{l_{1}}$ and $\Gamma_{l_{2}}$ we use the relation $b_{M+m}=(-1)^{M-1} b_{m}$. If all invariances $(1 \leq l \leq M-1)$ are used then we get an extended version of (19)

$$
\Gamma_{E_{1}} \mathbf{B} \Omega=\Gamma_{E_{2}} \mathbf{B}
$$

where $\Gamma_{E_{1}}$ and $\Gamma_{E_{2}}$ are formed by vertically staking $\Gamma_{l_{1}}$ and $\Gamma_{l_{2}}$ (for $1 \leq$ $l \leq M-1)$, respectively. Following the same procedure as in the previous section, we get

$$
\Psi=\left[\Gamma_{E_{1}} E_{s}\right]^{\dagger} \Gamma_{E_{2}} E_{s}=\left(E_{s}^{H} G_{1} E_{s}\right)^{-1}\left(E_{s}^{H} G_{2} E_{s}\right) .
$$


Here, $G_{1}=\left[\Gamma_{E_{1}}\right]^{H} \Gamma_{E_{1}}$ and $G_{2}=\left[\Gamma_{E_{1}}\right]^{H} \Gamma_{E_{2}}$ are $M \times M$ matrices that can be calculated off line. This means that in real time, the computational complexity of MBMP method will be same as BMP method and the required parameters can be obtained from the eigenvalues of $\Psi$.

In reduced dimensions if we select a subset of $k$ rows of $W^{H}$ then reduced dimensional selection matrices $\Gamma_{l 1}^{r}$ and $\Gamma_{l 2}^{r}$ of size $(k-l) \times k$ are appropriate sub blocks of $\Gamma_{l 1}$ and $\Gamma_{l 2}$, respectively. Now, the matrix $\Psi$ can be obtained as $\Psi=\left[\Gamma_{E_{1}}^{r} E_{s}^{r}\right]^{\dagger} \Gamma_{E_{2}}^{r} E_{s}^{r}$ where $\Gamma_{E_{1}}^{r}$ and $\Gamma_{E_{2}}^{r}$ are formed by vertically staking $\Gamma_{l 1}^{r}$ and $\Gamma_{l 2}^{r}($ for $1 \leq l \leq k-1)$, respectively.

\section{Computational complexity}

The most computationally demanding step in UMP, BMP and MBMP methods is to estimate signal subspace. After negligible computations, the complex data matrix $Y$ is transformed into a real matrix of size $M \times 2 L$ in UMP method and to calculate its left singular vectors, $8 M^{3}+8 M^{2} L$ real multiplications are required [6].

In beamspace methods this step can be divided into two sub-steps. In the first sub-step, $k$ rows of $W^{H}$ are multiplied with $Y$ to obtain $Y_{w}$. In the second sub-step the SVD of a real matrix of size $k \times 4 L$ is computed. Total multiplications require in these steps are $8 k^{3}+16 k^{2} L+8 k M L$. In BMP method, normally $k \ll M$, so the computational complexity of BMP method is several times less than the UMP method. In MBMP method, we suggest a larger value of $k$ to exploit the benefit of multiple-invariance. We propose $\frac{M}{3} \leq k \leq \frac{M}{2}$ then the computational burden of this step in MBMP method is typically $\frac{1}{2}$ to $\frac{1}{3}$ of UMP method. The computational burden of other steps is negligible and therefore not discussed, here.

\section{Simulation results}

Two closely spaced signals with $\theta_{1}=70^{\circ}$ and $\theta_{2}=75^{\circ}$ are impinging on a ULA of 10 sensors. Here we assume that $d=\frac{\lambda}{2}, L=3, M=8$ and $R_{1}=R_{2}=1$. We define the average root mean square error (RMSE) of estimates as

$$
\mathrm{RMSE}=\frac{\sqrt{E\left(\theta_{1}-\hat{\theta}_{1}\right)^{2}}+\sqrt{E\left(\theta_{2}-\hat{\theta}_{2}\right)^{2}}}{2}
$$

where $\hat{\theta}_{1}$ and $\hat{\theta}_{2}$ are estimates of $\theta_{1}$ and $\theta_{2}$ respectively. In Fig. 1 and 2 , RMSE of both estimates is plotted (in $\mathrm{dB}$ ) versus signal to noise ratio (SNR) which is defined as $\mathrm{SNR}=10 \log \left(1 / \sigma_{n}^{2}\right)$ where $\sigma_{n}^{2}$ is the variance of $\nu(n)$. The Cramer-Rao Lower Bound (CRLB) of the estimators is also calculated as in [2] and shown in these figures. Fig. 1 shows the comparison between BMP and MBMP methods when all beams are selected i.e. $k=M$. From this figure it is evident that MBMP method has a clear advantage over BMP method. The performance of UMP method is not shown here because when $k=M$ the results of MBMP and UMP methods are identical.

Fig. 2 shows the performance comparison of reduced dimension BMP (when $k=3$ and $k=\frac{M}{2}$ ) and MBMP (when $k=\frac{M}{2}$ ) methods with the 


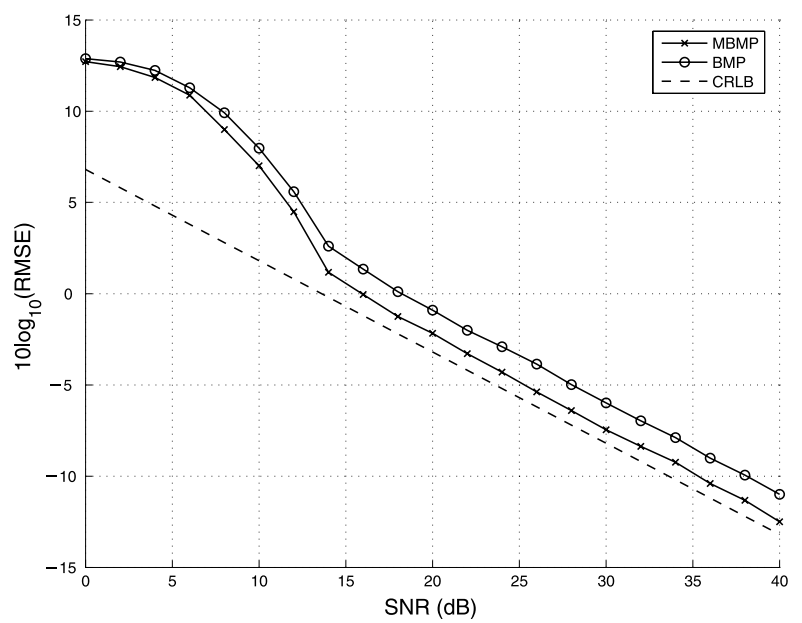

Fig. 1. Performance comparison of BMP and MBMP methods when all beams selected.

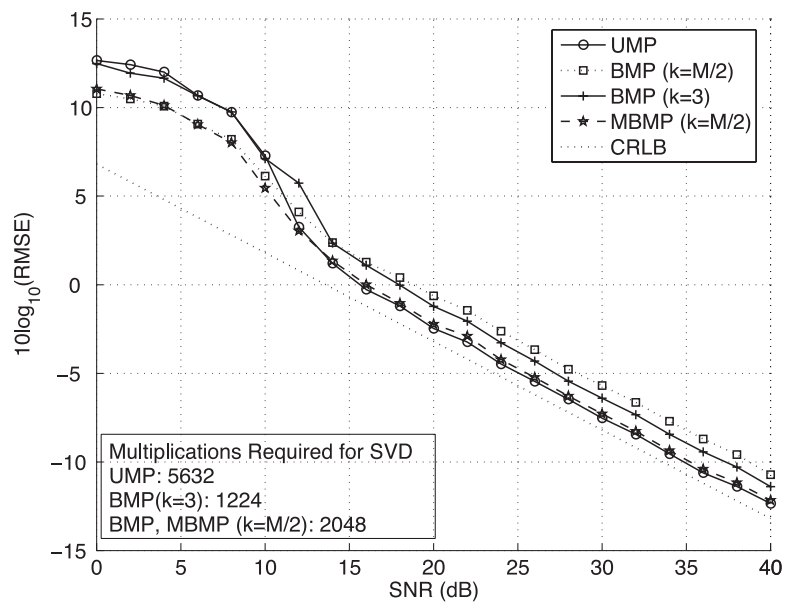

Fig. 2. Performance comparison of reduced BMP and MBMP methods with UMP method.

UMP method. The real multiplications required (for SVD) in each method are also shown in this figure. For low SNRs, the performance of BMP $(k=3)$ and MBMP methods is better than that of the UMP method. However, when SNR is increased the performance of BMP is slightly degraded but the performance of MBMP is almost equal to UMP method. Moreover, it can be easily observed that increasing $k$ does not improve BMP method's estimation accuracy.

\section{Conclusion}

In this paper, two novel BMP and MBMP methods are developed for DOA estimation. In situations (such as radar applications), where some prior information about DOAs is available, the reduced BMP and MBMP methods give improved performance (at low SNRs) with lesser computations as compared to the UMP method. At high SNRs, the performance of BMP method is somewhat degraded which can be improved by exploiting the multiple-invariance structure in the DFT transformed manifold vector using the MBMP method. 